\title{
Synthesis, antiprotozoal and antibacterial activity of nitro- and halogeno-substituted benzimidazole derivatives ${ }^{\star \star}$
}

\author{
Zygmunt Kazimierczuk ${ }^{1}{ }^{凶}$, Jacqueline A. Upcroft ${ }^{2}$, Peter Upcroft ${ }^{2}$, Agata Górska ${ }^{1}$, \\ Bohdan Starościak ${ }^{3}$ and Agnieszka Laudy ${ }^{3}$
}

${ }^{1}$ Institute of Chemistry, Agricultural University, Warszawa, Poland; ${ }^{2}$ The Queensland Institute of Medical Research, Brisbane, Australia; ${ }^{3}$ Medical University, Department of Pharmaceutical Microbiology, Warszawa, Poland

Received: 10 September, 2001; revised: 21 January, 2002; aceppted: 2 February, 2002

Key words: benzimidazoles, antiprotozoal activity, antibacterial activity

Two series of benzimidazole derivatives were sythesised. The first one was based on 5,6-dinitrobenzimidazole, the second one comprises 2-thioalkyl- and thioaryl-substituted modified benzimidazoles. Antibacterial and antiprotozoal activity of the newly obtained compounds was studied. Some thioalkyl derivatives showed remarkable activity against nosocomial strains of Stenotrophomonas malthophilia, and an activity comparable to that of metronidazole against Gram-positive and Gram-negative bacteria. Of the tested compounds, 5,6-dichloro-2-(4-nitrobenzylthio)-benzimidazole showed the most distinct antiprotozoal activity.

Benzimidazole derivatives are of wide interest because of their diverse biological activity and clinical applications [1]. This ring system is present in numerous antiparasitic, fungicidal, anithelemintic and anti-inflammatory drugs [2-5]. Also, some benzimidazole nucleosides, particularly 5,6-dichlorobenzimi- dazole-1- $\beta$-D-ribofuranoside (DRB) and its 2 -substituted derivatives show activity against human cytomegalovirus [6]. It is also known that 5,6-dinitrobenzimidazole can substitute 5,6-dimethylbenzimidazole in the vitamin $\mathrm{B}_{12}$ molecule in Corynebacterium diphteriae [7] and 2-trifluorobenzimidazoles are

\footnotetext{
${ }^{\star}$ Presented at the 8th International Symposium on Molecular Aspects of Chemotherapy, September, 2001, Gdańsk, Poland.

${ }^{\star}$ We would like to thank the National Health and Medical Research Council of Australia for their support. The study was in part supported by The Foundation for Development of Diagnostics and Therapy, Warsaw, Poland.

${ }^{\circledR}$ Corresponding author: Zygmunt Kazimierczuk, Agricultural University, Institute of Chemistry, Nowoursynowska 159C, 02-787 Warszawa, Poland; e-mail: kazimierczuk@delta.sggw.waw.pl

Abbreviations: CFU, colony-forming unit; DBU, 1,8-diazabicyclo[5,4,0]undecen-7-en; DRB, 5,6-dichlorobenzimidazole-1- $\beta$-D-ribofuranoside; $\mathrm{Me}_{2} \mathrm{SO}$, dimethylsulfoxide; $\mathrm{MIC}$, minimal inhibitory concentration.
} 
potent decouplers of oxidative phosphorylation in mitochondria. They are also inhibitors of photosynthesis, and some exhibit appreciable herbicidal activity [8]. Most recently, antiprotozoal activity of substituted 2-trifluorobenzimidazoles has been reported [9], consistent with several earlier studies on the anti-giardial activity of various benzimidazole derivatives [10, 11]. However, the general antimicrobial activity of benzimidazole derivatives has not been extensively investigated. The earliest report of their antibacterial activity appeared in 1964 [12], and more recently we have found two groups of substituted benzimidazoles, namely the 5,6-dinitro and 2-trifluoromethyl derivatives, to be promising candidates for antimicrobial drugs [13]. In this paper we present new data on the antimicrobial and antiprotozoal activities of 5,6-dinitro and 2-dialkylaminosubstituted benzimidazoles.

\section{MATERIALS AND METHODS}

General methods. All chemicals and solvents were purchased from Sigma-Aldrich. Melting points (uncorr.) were measured in open capillary tubes on a Gallenkamp-5 melting point apparatus. Ultraviolet absorption spectra were recorded in a Kontron Uvikon 940 spectrophotometer. ${ }^{1} \mathrm{H}-\mathrm{NMR}$ spectra (in ppm) were measured with a Varian Gemini (200 MHz) and a Varian UNITY plus (500 $\mathrm{MHz}$ ) spectrometer at $298 \mathrm{~K}$ in $\mathrm{D}_{6}\left(\mathrm{Me}_{2} \mathrm{SO}\right)$ using tetramethylsilane as internal standard. Flash chromatography was performed with Merck silica gel 60 (200-400 mesh). Analytical TLC was carried out on precoated silica gel $\mathrm{F}_{254}$ (Merck) plates (0.25 mm thickness). Analyses of the new compounds were within $\pm 0.4 \%$ of the theoretical values.

Bacterial strains and drug susceptibility assays. Bacteria were used as follows: Staphylococcus aureus strains ATCC 6538P, ATCC 25923 and NCTC 4163; Bacillus subtilis ATCC 6633, Bacillus stearothermophilus ATCC 7953 and Bacillus cereus ML 98; Escherichia coli NCTC 8196; Proteus vulgaris NCTC 4635; Micrococcus flavus NCIB 8166.

Bacterial strains from Pseudomonadaceae family: Pseudomonas aeruginosa ATCC 27863 , ATCC 15442 and NCTC 6749; Burkholderia cepacia ATCC 25416; Stenotrophomonas malthophilia ATCC 13637 and clinical isolates -67 strains of $S$. malthophilia were used. Bacterial strains were obtained from the State Institute of Hygiene (Warszawa), the Medical University, Warszawa and the Children's Memorial Health Institute.

Antimicrobial activity was tested by the disc-diffusion method under standard conditions using Mueller-Hinton agar medium as described by NCCLS [14]. Sterile filter paper discs (9 mm diameter, Whatman No. 3 chromatography paper) were soaked in test compound solutions prepared in $\mathrm{EtOH} / \mathrm{Me}_{2} \mathrm{SO}$ mixture (1:1, $\mathrm{v} / \mathrm{v})$ and finally contained $400 \mu \mathrm{g}$ of the compound per disc. The results were read following $18-20 \mathrm{~h}$ incubation at $35^{\circ} \mathrm{C}$. MIC - the minimum inhibitory concentration - was performed by the agar dilution method according to guidelines established by the NCCLS [15]. MIC was described as the lowest concentration of the drug that visibly inhibited growth. A single colony or faint haze was regarded as no growth. MICs were interpreted after 18 and $42 \mathrm{~h}$ of incubation at $35^{\circ} \mathrm{C}$. Concentrations of agents in MullerHinton II Agar medium (Becton Dickinson) ranged from 12.5 to $400 \mu \mathrm{g} \times \mathrm{cm}^{-3}$. Agar plates were inoculated using a replicator with $2 \mu \mathrm{l}$ volumes. The final inoculum of bacteria was $10^{-4} \mathrm{CFU} \times \mathrm{cm}^{-3}$. Solutions of the agents tested were prepared in $0.125 \mathrm{M} \mathrm{HCl}$, for compounds $\mathbf{4 d}$, $\mathbf{4 f}$ and $\mathbf{4 n}$, and in a 0.125 $\mathrm{M} \mathrm{HCl} / \mathrm{MeOH}$ mixture (1:0.6, v/v) for compound $4 \mathbf{e}$.

Protozoan parasite isolates, culture and drug susceptibility assays. Giardia duodenalis, Trichomonas vaginalis and Entamoeba histolytica were grown in TYI-S-33 which was supplemented with bile for Giardia [16]. Parasites were subcultured three times a week ex- 
cept for Entamoeba which was subcultured twice a week [16]. T. vaginalis strain BRIS/ 92/STDL/F1623 and the metronidazole resistant line derived from it BRIS/92/STDL/ F1623-M1 (F1623-M1), E. histolytica strain HTH-56:MUTM (MUTM) and G. duodenalis strains BRIS/83/HEPU/ 106 (referred to as 106), the metronidazole-resistant line BRIS/83/HEPU/106-2ID 10 derived from 106 $\left(106-2 \mathrm{ID}_{10}\right), \mathrm{WB} 1 \mathrm{~B}$, the metronidazole-resistant line WB-M3 and the albendazole resistant line WB-M3-Alb (WB/Alb) are described in [16]. Drug susceptibility assays were detailed previously [16].

\section{Syntheses}

1-Substituted 5,6-dinitrobenzimidazoles. 5,6-Dinitro-1-(dimethylaminoeth-1-yl)benzimidazole (2a): $1.05 \mathrm{~g}$ (5 mmol) of 5,6-dinitrobenzimidazole (1) was dissolved in acetonitrile (40 ml) containing $2.4 \mathrm{ml}$ (16 mmol) 1,8-diazabicyclo[5,4,0]undecen-7-en (DBU) followed by addition of $0.864 \mathrm{~g}$ ( $6 \mathrm{mmol})$ of 2-dimethylaminoethylchloride hydrochloride. The solution was stirred overnight at room temperature and then refluxed for $1 \mathrm{~h}$. The reaction mixture was evaporated to dryness and the residue was chromatographed on a silica gel column $(3 \times 15 \mathrm{~cm})$ with $\mathrm{CHCl}_{3}(200 \mathrm{ml})$, followed by $\mathrm{CHCl}_{3} / \mathrm{MeOH}(95: 5)$. The product-containing fractions were evaporated and the residue crystallized from ethyl acetate $/ \mathrm{MeOH}$ to give $2 \mathbf{a}(0.90$ g, 64\%); m.p. $173-177^{\circ} \mathrm{C} ;{ }^{1} \mathrm{H}$ NMR $\left(\mathrm{D}_{6}-\mathrm{Me}_{2} \mathrm{SO}\right) \delta$ (ppm): $2.16\left(\mathrm{~s}, 2 \times \mathrm{CH}_{3}\right), 2.65$ and $4.49(2 \times \mathrm{t}$, $-\mathrm{CH}_{2} \mathrm{CH}_{2}$ ) $), 8.53,8.72$ and $8.75(3 \mathrm{~s}, \mathrm{H}-2, \mathrm{H}-4$ and $\mathrm{H}-7)$. UV $\left(\mathrm{H}_{2} \mathrm{O} / \mathrm{MeOH}, 1: 1\right): 246 \mathrm{~nm}$ (16900), 300 (sh, 6000). Elemental analysis: calculated for $\mathrm{C}_{11} \mathrm{H}_{13} \mathrm{~N}_{5} \mathrm{O}_{4}$ : C, 47.30; $\mathrm{H}, 4.70$; N, 25.08. Found: C, 47.47; H, 4.61; N, 25.07.

5,6-Dinitro-1-(diethylaminoeth-1-yl)benzimidazole (2b): Compound $\mathbf{2 b}$ was similarly synthesised to the procedure described above for $\mathbf{2 a}$, from $\mathbf{1}$ and 2-diethylaminoethylchloride hydrochloride: (m.p. $123-127^{\circ} \mathrm{C}, 49 \%$ ); ${ }^{1} \mathrm{H}$
NMR ( $\left.\mathrm{D}_{6}-\mathrm{Me}_{2} \mathrm{SO}\right): \delta$ (ppm): 0.71 and $2.42(\mathrm{t}$ and $\left.\mathrm{q}, \mathrm{N}-\mathrm{CH}_{2} \mathrm{CH}_{3}\right), 2.71$ and $4.44(2 \times \mathrm{t}$, $-\mathrm{CH}_{2} \mathrm{CH}_{2}$ ), 8.52, 8.70 and $8.73(3 \mathrm{~s}, \mathrm{H}-2, \mathrm{H}-4$, $\mathrm{H}-7)$. UV ( $\left.\mathrm{H}_{2} \mathrm{O} / \mathrm{MeOH}, 1: 1\right): 246 \mathrm{~nm}$ (18200), 300 (sh, 6000). Elemental analysis: calculated for $\mathrm{C}_{13} \mathrm{H}_{17} \mathrm{~N}_{5} \mathrm{O}_{4}$ : C, 50.80; H, 5.59; N, 22.79. Found: C, 50.92; H, 6.05; N, 22.62.

5,6-Dinitro-1-(dimethylaminoprop-1-yl)benzimidazole (2c): The synthesis was similar to the procedure for $\mathbf{2 a}$, from $\mathbf{1}$ and 3-dimethylaminopropylchloride hydrochloride: (m.p. $\left.232-235^{\circ} \mathrm{C}, 38 \%\right) ;{ }^{1} \mathrm{H}$ NMR $\left(\mathrm{D}_{6}-\mathrm{Me}_{2} \mathrm{SO}\right): \delta$ (ppm): $2.70\left(\mathrm{~s}, 2 \times \mathrm{CH}_{3}\right), 2.24,3.03$ and 4.53 ( $2 \times \mathrm{t}$ and $\mathrm{m},-\mathrm{CH}_{2} \mathrm{CH}_{2} \mathrm{CH}_{2}$-), 8.59, 8.79 and 8.87 (3s, $\mathrm{H}-2, \mathrm{H}-4, \mathrm{H}-7)$. UV ( $\left.\mathrm{H}_{2} \mathrm{O} / \mathrm{MeOH}, 1: 1\right)$ : $246 \mathrm{~nm}$ (18200), 300 (sh, 6000). Elemental analysis: calculated for $\mathrm{C}_{12} \mathrm{H}_{15} \mathrm{~N}_{5} \mathrm{O}_{4}$ : C, 49.13; H, 5.17; N, 23.88. Found: C, 49.30; H, 5.19; N, 22.62.

5,6-Dinitro-1-(morpholineth-1-yl)benzimidazole (2d): Similar to $\mathbf{2 a}$, from $\mathbf{1}$ and $N$ (2-chloroeth-1-yl)morpholine hydrochloride: (m.p. $\left.166-169^{\circ} \mathrm{C}, 42 \%\right) ;{ }^{1} \mathrm{H}$ NMR $\left(\mathrm{D}_{6}-\mathrm{Me}_{2} \mathrm{SO}\right)$ $\delta$ (ppm): 2.69 and $4.54\left(2 \times \mathrm{t},-\mathrm{CH}_{2} \mathrm{CH}_{2}\right.$-), 2.42 and $3.48\left(2 \times \mathrm{t}, \mathrm{CH}_{2}\right.$-morpholine), 8.51, 8.71 and 8.75 (3s, H-2, H-4 and H-7). UV $\left(\mathrm{H}_{2} \mathrm{O} / \mathrm{MeOH}, 1: 1\right): 247 \mathrm{~nm}$ (18500), 310 (sh, 6000). Elemental analysis: calculated for $\mathrm{C}_{13} \mathrm{H}_{15} \mathrm{~N}_{5} \mathrm{O}_{5}$ : C, 48.59; $\mathrm{H}, 4.71 ; \mathrm{N}, 21.80$. Found: C, 48.69; H, 4.86; N, 21.60.

5,6-Dinitro-1-(piperidineth-1-yl)benzimidazole (2e): Similar to $\mathbf{2 a}$, from $\mathbf{1}$ and $N$-(2-chloroeth-1-yl)piperidine hydrochloride: (m.p. $\left.206-208^{\circ} \mathrm{C}, 26 \%\right) ;{ }^{1} \mathrm{H}$ NMR $\left(\mathrm{D}_{6}-\mathrm{Me}_{2} \mathrm{SO}\right) \delta$ (ppm): 1.38 (m, $\mathrm{CH}_{2}$-piperidine), 2.63 and 4.49 ( $2 \times \mathrm{t},-\mathrm{CH}_{2} \mathrm{CH}_{2}$ ) $), 8.53,8.71$ and $8.73(3 \mathrm{~s}, \mathrm{H}-2$, H-4 and H-7). UV ( $\left.\mathrm{H}_{2} \mathrm{O} / \mathrm{MeOH}, 1: 1\right): 246 \mathrm{~nm}$ (17200), 300 (sh, 6000). Elemental analysis: calculated for $\mathrm{C}_{14} \mathrm{H}_{17} \mathrm{~N}_{5} \mathrm{O}_{4}$ : C, 52.65; H, 5.38; N, 21.93. Found: C, 52.83; H, 5.52; N, 21.77. 5,6-Dinitro-1-(2-hydroxyeth-1-yl)benzimidazole (2f): Similar to $\mathbf{2 a}$, from $\mathbf{1}$ and 2-bromoethanol: (m.p. $235-238^{\circ} \mathrm{C}, 56 \%$ ); ${ }^{1} \mathrm{H}$ NMR $\left(\mathrm{D}_{6}-\mathrm{Me}_{2} \mathrm{SO}\right) \delta$ (ppm): 3.75 and 4.46 (q and $\mathrm{t}$, $-\mathrm{CH}_{2} \mathrm{CH}_{2}$ ), 8.53, 8.66 and 8.72 (3s, H-2, H-4 and $\mathrm{H}-7)$. UV $\left(\mathrm{H}_{2} \mathrm{O} / \mathrm{MeOH}, 1: 1\right): 247 \mathrm{~nm}$ 
(16500), 300 (sh, 6000). Elemental analysis: calculated for $\mathrm{C}_{9} \mathrm{H}_{8} \mathrm{~N}_{5} \mathrm{O}_{5}: \mathrm{C}, 42.86 ; \mathrm{H}, 3.20 ; \mathrm{N}$, 22.22. Found: C, 42.99; H, 3.34; N, 22.01.

5,6-Dinitro-1-(2,3-dihydroxyprop-1-yl)benzimidazole ( $\mathbf{2} \mathbf{g})$ : Similar to $\mathbf{2 a}$, from $\mathbf{1}$ and glycitole: (m.p. $198-201^{\circ} \mathrm{C}, 47 \%$ ); ${ }^{1} \mathrm{H}$ NMR $\left(\mathrm{D}_{6}-\mathrm{Me}_{2} \mathrm{SO}\right) \delta$ (ppm): $3.32\left(\mathrm{~m}, \mathrm{CH}_{2}-3^{\prime}\right), 4.35$ (m, CH-2'), 4.53 (dd, $\mathrm{CH}_{2}$ - $^{\prime}$ ), 4.91 (t, HO-3'), $5.19\left(\mathrm{~d}, \mathrm{HO}-2^{\prime}\right), 8.53,8.61$ and $8.62(3 \mathrm{~s}, \mathrm{H}-2$, $\mathrm{H}-4$ and $\mathrm{H}-7)$; UV $\left(\mathrm{H}_{2} \mathrm{O} / \mathrm{MeOH}, 1: 1\right): 249 \mathrm{~nm}$ (17200), 300 (sh, 7000). Elemental analysis: calculated for $\mathrm{C}_{10} \mathrm{H}_{10} \mathrm{~N}_{4} \mathrm{O}_{6}$ : $\mathrm{C}, 42.55 ; \mathrm{H}, 3.58$; N, 19.86. Found: C, 42.33; H, 3.45; N, 19,80.

S-alkylated and -arylalkylated derivatives of C-substituted benzimidazoles (4a-m). 2-(Dimethylaminoeth-1-ylthio)benzimidazole (4a): $0.6 \mathrm{~g}$ (4 mmol) of 2-thiobenzimidazole was dissolved in acetonitrile (20 $\mathrm{ml}$ ) containing $2 \mathrm{ml}$ (14 mmol) DBU followed by the addition of $0.72 \mathrm{~g}(5 \mathrm{mmol})$ of 2-dimethylaminoethylchloride hydrochloride. The solution was stirred overnight at room temperature and the solvent evaporated. Water $(5 \mathrm{ml})$ was added to the residue and the mixture was brought to $\mathrm{pH} 7$ with $\mathrm{CH}_{3} \mathrm{COOH}$. The precipitate formed was filtered and crystallized from $\mathrm{H}_{2} \mathrm{O}$ to give $\mathbf{4 a}(0.424 \mathrm{~g}, 48 \%)$; m.p. $137-140^{\circ} \mathrm{C} ;{ }^{1} \mathrm{H}$ NMR $\left(\mathrm{D}_{6}-\mathrm{Me}_{2} \mathrm{SO}\right) \delta$ (ppm): 2.41 (s, $2 \times \mathrm{CH}_{3}$ ), 2.88 and 3.47 (2t, $-\mathrm{CH}_{2} \mathrm{CH}_{2}$ ), 7.10 and 7.44 (2m, H-benzimidazole). UV ( $\mathrm{H}_{2} \mathrm{O}, \mathrm{pH}$ 7.0): 282 (10 500), 289 (10300). Elemental analysis: calculated for $\mathrm{C}_{11} \mathrm{H}_{15} \mathrm{~N}_{3} \mathrm{~S}: \mathrm{C}, 59.70 ; \mathrm{H}, 6.85 ; \mathrm{N}, 18.99$. Found: C, 59.55; H, 6.66: N, 18.83.

5,6-Dimethyl-2-(dimethylaminoeth-1-ylthio)benzimidazole (4b): Similar to $\mathbf{4 a}$, from $\mathbf{3 b}$ and 2-dimethylaminoethylchloride hydrochloride. The crude product was purified by silica gel column chromatography $(3 \times 15 \mathrm{~cm})$ using $\mathrm{CHCl}_{3} / \mathrm{MeOH}$ (85:15) as an eluent. Crystallization from diethyl ether/petroleum ether yielded 4b (340 mg, 39\%); m.p. $135-137^{\circ} \mathrm{C} ;{ }^{1} \mathrm{H}$ $\operatorname{NMR}\left(\mathrm{D}_{6}-\mathrm{Me}_{2} \mathrm{SO}\right) \delta(\mathrm{ppm}): 2.18$ and $2.26(2 \mathrm{~s}$, $2 \times \mathrm{CH}_{3}$-benzimidazole and $\left.2 \times \mathrm{N}_{-} \mathrm{CH}_{3}\right), 2.57$ and 3.35 (2t, $-\mathrm{CH}_{2} \mathrm{CH}_{2}$-), 7.19 (s, H-4 and H-7). UV, ( $\mathrm{H}_{2} \mathrm{O}, \mathrm{pH}$ 7.0): 291 (13500), 297 (13 300). Elemental analysis: calculated for $\mathrm{C}_{13} \mathrm{H}_{19}$ -
$\mathrm{N}_{3} \mathrm{~S}: \mathrm{C}, 62.61 ; \mathrm{H}, 7.70 ; \mathrm{N}, 16.86$. Found: C, $62.86 ; \mathrm{H}, 7.55 ; \mathrm{N}, 16.88$.

5-Chloro-2-(dimethylaminoeth-1-ylthio)benzimidazole hydrochloride (4c): Similar to $\mathbf{4 b}$, from 3c and 2-dimethylaminoethylchloride hydrochloride. Pure product was treated with $\mathrm{MeOH}$ saturated with dry $\mathrm{HCl}$ to give $4 \mathbf{c}$ (m.p. $\left.239-242^{\circ} \mathrm{C} \quad 42 \%\right) ;{ }^{1} \mathrm{H}$ NMR $\left(\mathrm{D}_{6}-\mathrm{Me}_{2} \mathrm{SO}\right) \delta$ (ppm): 2.83 (s, $2 \times \mathrm{CH}_{3}$ ), 3.48 and $3.74(2 \mathrm{t}$, $-\mathrm{CH}_{2} \mathrm{CH}_{2}$ ), 7.26 and 7.54 (2dd, H-6 and H-7), 7.61 (d, H-4). UV ( $\left.\mathrm{H}_{2} \mathrm{O}, \mathrm{pH} 7.0\right): 251$ (5600), 290 (12500), 298 (12300). Elemental analysis: calculated for $\mathrm{C}_{11} \mathrm{H}_{14} \mathrm{~N}_{3} \mathrm{SCl} \cdot \mathrm{HCl}$ : C, 45.21; H, 5.18; N, 14.38. Found: C, 45.12; H, 5.27 ; N, 14.50 .

5, 6-Dichloro-2-(dimethylaminoeth 1-ylthio)benzimidazole hydrochloride (4d): Similar to 4a, from $\mathbf{3 d}$ and 2-dimethylaminoethylchloride hydrochloride and transformed to hydrochloride analogously to the method given for $\mathbf{4 c}$ (m.p. $\left.193-195^{\circ} \mathrm{C}, 44 \%\right) ;{ }^{1} \mathrm{H} \mathrm{NMR}\left(\mathrm{D}_{6}-\mathrm{Me}_{2} \mathrm{SO}\right)$ $\delta$ (ppm): 2.82 (s, $2 \times \mathrm{CH}_{3}$ ), 3.43 and 3.66 (2t, $-\mathrm{CH}_{2} \mathrm{CH}_{2}$ ), 7.73 (s, $\mathrm{H}-4$ and $\left.\mathrm{H}-7\right)$. UV $\left(\mathrm{H}_{2} \mathrm{O}, \mathrm{pH}\right.$ 7.0): 256 (6300), 297 (12900), 306 (13300). Elemental analysis: calculated for $\mathrm{C}_{11} \mathrm{H}_{13} \mathrm{~N}_{3}$ $\mathrm{Cl}_{2} \mathrm{~S} \cdot \mathrm{HCl}: \mathrm{C}, 40.44 ; \mathrm{H}, 4.33 ; \mathrm{N}, 12.86$. Found: C, 40.31; H, 4.52; N, 12,73.

5,6-Dichloro-2-(diethylaminoeth-1-ylthio)benzimidazole (4e): Similar to $\mathbf{4 a}$, from $\mathbf{3 d}$ and 2-diethylaminoethylchloride hydrochloride: (m.p. $266-269^{\circ} \mathrm{C}$, from $\left.\mathrm{H}_{2} \mathrm{O} / \mathrm{EtOH}, 81 \%\right) ;{ }^{1} \mathrm{H}$ NMR $\left(\mathrm{D}_{6}-\mathrm{Me}_{2} \mathrm{SO}\right) \delta(\mathrm{ppm}): 1.26$ and $3.18(\mathrm{t}$ and $\mathrm{q}, 2 \times \mathrm{C}_{2} \mathrm{H}_{5}$ ), 3.35 and 3.61 (2t, $-\mathrm{CH}_{2} \mathrm{CH}_{2}$ ), 7.73 (s, H-4 and $\left.\mathrm{H}-7\right)$. UV ( $\mathrm{H}_{2} \mathrm{O}, \mathrm{pH}$ 7.0): 256 (5800), 291 (13000), 297 (13300). Elemental analysis: calculated for $\mathrm{C}_{13} \mathrm{H}_{17^{-}}$ $\mathrm{N}_{3} \mathrm{Cl}_{2} \mathrm{~S}$ : C, 49.06; H, 5.40; N, 13.21. Found: C, 49.30; H, 5.54; N, 13.43.

5,6-Dichloro-2-(dimethylaminoprop-1-ylthio)benzimidazole hydrochloride (4f): Similar to 4a, from 3d and 3-dimethylaminopropylchloride hydrochloride. The crude product was purified by column chromatography (silica gel, $3 \times 15 \mathrm{~cm}$ ) using $\mathrm{CHCl}_{3} / \mathrm{MeOH}$ (80:20) as an eluent. The product-containing fractions were evaporated and the product was transformed to the hydrochloride as de- 
scribed for 4c: (m.p. $\left.219-222^{\circ} \mathrm{C}, 56 \%\right) ;{ }^{1} \mathrm{H}$ NMR $\left(\mathrm{D}_{6}-\mathrm{Me}_{2} \mathrm{SO}\right) \delta(\mathrm{ppm}): 2.51\left(\mathrm{~s}, 2 \times \mathrm{CH}_{3}\right)$, 2.15, 3.16 and 3.44 (2t and $\mathrm{q},-\mathrm{CH}_{2} \mathrm{CH}_{2} \mathrm{CH}_{2}$-), 7.76 (s, $\mathrm{H}-4$ and $\mathrm{H}-7)$. UV ( $\mathrm{H}_{2} \mathrm{O}, \mathrm{pH}$ 7.0): 256 (6 000), 297 (13100), 306 (13400). Elemental analysis: calculated for $\mathrm{C}_{12} \mathrm{H}_{15} \mathrm{~N}_{3} \mathrm{Cl}_{2} \mathrm{~S} \cdot \mathrm{HCl}$ : C, 42.31; H, 4.74; N, 12.34. Found: C, 42.44; $\mathrm{H}, 4.91 ; \mathrm{N}, 12.48$.

5,6-Dichloro-2-(morpholineth-1-yl)benzimidazole hydrochloride (4g): Similar to $\mathbf{4 a}$, from 3d and morpholineethyl-chloride hydrochloride. The crude product was purified by column chromatography as described for $\boldsymbol{4 f}$ and transformed into the hydrochloride as described for 4c: (m.p. $\left.147-150^{\circ} \mathrm{C}, 55 \%\right) ;{ }^{1} \mathrm{H}$ NMR ( $\left.\mathrm{D}_{6}-\mathrm{Me}_{2} \mathrm{SO}\right) \delta$ (ppm): 3.20 and 3.73 (bs and $\mathrm{t},-\mathrm{CH}_{2} \mathrm{CH}_{2}$ ), 3.52 and 3.92 ( $\mathrm{t}$ and $\mathrm{bs}$, $\mathrm{CH}_{2}$-morpholine), 7.73 (s, $\mathrm{H}-4$ and $\mathrm{H}-7$ ). UV ( $\mathrm{H}_{2} \mathrm{O}, \mathrm{pH}$ 7.0): 256 (5900), 297 (13200), 306 (13500). Elemental analysis: calculated for $\mathrm{C}_{13} \mathrm{H}_{15} \mathrm{~N}_{3} \mathrm{Cl}_{2} \mathrm{~S} \cdot \mathrm{HCl}: \mathrm{C}, 42.35 ; \mathrm{H}, 4.38 ; \mathrm{N}$, 11.40. Found: C, 42.46; H, 4.52; N, 11.27.

5,6-Dichloro-2-(4-nitrobenzylthio)benzimidazole (4h): Similar to $\mathbf{4 a}$, from $\mathbf{3 d}$ and 4-nitrobenzyl chloride: (m.p. $134-138^{\circ} \mathrm{C}$, from $\mathrm{EtOH} / \mathrm{H}_{2} \mathrm{O}$, 59\%); ${ }^{1} \mathrm{H}$ NMR $\left(\mathrm{D}_{6}-\mathrm{Me}_{2} \mathrm{SO}\right) \delta$ (ppm): 4.70 (s, $\mathrm{CH}_{2}$ ), 7.60-8.10 (2m, H-arom. and H-benzimidazole). $\mathrm{UV}\left(\mathrm{H}_{2} \mathrm{O} / \mathrm{MeOH}, 1: 1\right)$ : 265 (13400), 302 (17 700), 308 (17 100). Elemental analysis: calculated for $\mathrm{C}_{14} \mathrm{H}_{9} \mathrm{~N}_{3}$ $\mathrm{O}_{2} \mathrm{Cl}_{2} \mathrm{~S}: \mathrm{C}, 47.48 ; \mathrm{H}, 2.57 ; \mathrm{N}, 11.87$. Found: $\mathrm{C}$, 47.40; H, 2.39; N, 11.70 .

5,6-Dichloro-2-(3, 4-dichlorobenzylthio)benzimidazole (4i): Similar to $\mathbf{4 a}$, from $\mathbf{3 d}$ and 3,4-dichlorobenzyl chloride: (m.p. $157-159^{\circ} \mathrm{C}$, from $\left.\mathrm{EtOH} / \mathrm{H}_{2} \mathrm{O}, 39 \%\right) ;{ }^{1} \mathrm{H} \mathrm{NMR}\left(\mathrm{D}_{6}-\mathrm{Me}_{2} \mathrm{SO}\right)$ $\delta$ (ppm): $4.56\left(\mathrm{~s}, \mathrm{CH}_{2}\right), 7.40-7.80$ (m, H-arom. and H-benzimidazole). UV $\left(\mathrm{H}_{2} \mathrm{O} / \mathrm{MeOH}, 1: 1\right)$ : 263 (7300), 303 (16800), 310 (17000). Elemental analysis: calculated for $\mathrm{C}_{14} \mathrm{H}_{8} \mathrm{~N}_{2} \mathrm{Cl}_{4} \mathrm{~S}$ : C, 44.48; H, 2.14; N, 7.41. Found: C, 44.63; H, $2.26 ; \mathrm{N}, 7.30$.

5-Carboxy-2-allylthiobenzimidazole (4j): Similar to $\mathbf{4 a}$, from $\mathbf{3 j}$ and allyl chloride using $\mathrm{NaOH}$ instead of DBU: (from $\mathrm{MeOH}, 72 \%$, m.p. $\left.112-115^{\circ} \mathrm{C}\right) ;{ }^{1} \mathrm{H}$ NMR $\left(\mathrm{D}_{6}-\mathrm{Me}_{2} \mathrm{SO}\right) \delta$ (ppm): 5.13 and $5.34\left(2 \mathrm{~d},=\mathrm{CH}_{2}\right), 6.00(\mathrm{~m}$,
$\mathrm{HC}=$ ), 7.52 and 7.78 (2dd, H-6 and H-7), 8.02 (s, H-4). UV ( $\left.\mathrm{H}_{2} \mathrm{O} / \mathrm{MeOH}, 9: 1\right): 225$ (24500), 299 (14400). Elemental analysis: calculated for $\mathrm{C}_{14} \mathrm{H}_{8} \mathrm{~N}_{2} \mathrm{Cl}_{4} \mathrm{~S} \cdot \mathrm{H}_{2} \mathrm{O}: \mathrm{C}, 52.37 ; \mathrm{H}, 4.80 ; \mathrm{N}$, 11.11. Found: C, 52.29; H, 4.58; N, 11.14.

5-Carboxy-2-benzylthiobenzimidazole (4k): Similar to $\mathbf{4 j}$, from $\mathbf{3 j}$ and benzyl chloride: (m.p. $131-134^{\circ} \mathrm{C}$, from $\mathrm{MeOH}, 64 \%$ ); ${ }^{1} \mathrm{H} \mathrm{NMR}$ (D $\left.\mathrm{D}_{6}-\mathrm{Me}_{2} \mathrm{SO}\right) \delta$ (ppm): $4.62\left(\mathrm{~s}, \mathrm{CH}_{2}\right.$ ), 7.30-7.60 (m, H-arom.), 7.48 and 7.82 (2dd, $\mathrm{H}-6$ and $\mathrm{H}-7), 8.03$ (s, H-4). UV ( $\left.\mathrm{H}_{2} \mathrm{O} / \mathrm{MeOH}, 9: 1\right): 224$ (29800), 300 (14 300). Elemental analysis: calculated for $\mathrm{C}_{14} \mathrm{H}_{8} \mathrm{~N}_{2} \mathrm{Cl}_{4} \mathrm{~S} \cdot \mathrm{H}_{2} \mathrm{O}: \mathrm{C}, 63.37 ; \mathrm{H}$, 4.26; N, 9.86. Found: C, 63.30; H, 4.40; N, 9.81 .

5-Carboxy-2-(4-nitrobenzylthio)benzimidazole (41): Similar to $\mathbf{4 j}$, from $\mathbf{3} \mathbf{j}$ and 4-nitrobenzyl chloride: (m.p. $283-286^{\circ} \mathrm{C}$, from $\mathrm{MeOH}, 68 \%$ ); ${ }^{1} \mathrm{H}$ NMR $\left(\mathrm{D}_{6}-\mathrm{Me}_{2} \mathrm{SO}\right) \delta$ (ppm): $4.69\left(\mathrm{~s}, \mathrm{CH}_{2}\right)$, 7.20-8.20 (m, H-arom. and H-benzimidazole). $\mathrm{UV}\left(\mathrm{H}_{2} \mathrm{O} / \mathrm{MeOH}, 1: 1\right): 294$ (18900). Elemental analysis: calculated for $\mathrm{C}_{15} \mathrm{H}_{11} \mathrm{~N}_{3} \mathrm{SO}_{4}$ : C, 54.71; H, 3.37; N, 12.76. Found: C, 54.89; H, $3.44 ; \mathrm{N}, 12.62$.

5-Carboxy-2-(3,4-dichlorobenzylthio)benzimidazole (4m): Similar to $\mathbf{4 j}$, from $\mathbf{3 j}$ and 3,4-dichlorobenzylchloride: (m.p. $284-288^{\circ} \mathrm{C}$, from $\mathrm{MeOH}, 73 \%) ;{ }^{1} \mathrm{H} \mathrm{NMR}\left(\mathrm{D}_{6}-\mathrm{Me}_{2} \mathrm{SO}\right) \delta$ (ppm): 4.64 (s, $\mathrm{CH}_{2}$ ), 7.40-7.85 (m, H-arom., $\mathrm{H}-6$ and $\mathrm{H}-7), 8.05$ (s, H-4). UV $\left(\mathrm{H}_{2} \mathrm{O} / \mathrm{MeOH}\right.$, 1:1): 229 (36600), 302 (15400). Elemental analysis: calculated for $\mathrm{C}_{15} \mathrm{H}_{10} \mathrm{~N}_{2} \mathrm{SCl}_{2} \mathrm{O}_{2}$. $\mathrm{H}_{2} \mathrm{O}: \mathrm{C}, 48.53 ; \mathrm{H}, 3.26 ; \mathrm{N}, 7.55$. Found: $\mathrm{C}$, 47.41; H, 3.08; N, 7.31.

4,6-Dichloro-2-thiobenzimidazole (6): $1.168 \mathrm{~g}$ (6.6 mmol) of 1,3-dichloro-4,5-diaminobenzene (5) was dissolved in a mixture of $\mathrm{EtOH}$ $(13 \mathrm{ml})$ and water $(2 \mathrm{ml})$, followed by the addition of $0.8 \mathrm{ml}$ (13.2 mmol) of carbon disulfide and $1.48 \mathrm{~g}$ (26.4 mmol) of $\mathrm{KOH}$. The solution was stirred under reflux for $2 \mathrm{~h}$, decolorized with charcoal and brought to $\mathrm{pH} 5$ with acetic acid. The yellowish chromatographic pure precipitate was removed by filtration. Yield $0.9 \mathrm{~g}(62 \%)$. A small amount for analysis was crystallized from water: (m.p. $\left.>300^{\circ} \mathrm{C}\right) .{ }^{1} \mathrm{H}$ $\operatorname{NMR}\left(\mathrm{D}_{6}-\mathrm{Me}_{2} \mathrm{SO}\right) \delta$ (ppm): 7.12 and 7.32 (2dd, 
$\mathrm{H}-5$ and $\mathrm{H}-7)$. UV ( $\mathrm{H}_{2} \mathrm{O}, \mathrm{pH}$ 7): 230 (12 100), 251 (16300), 314 (17900). Elemental analysis: calculated for $\mathrm{C}_{7} \mathrm{H}_{4} \mathrm{~N}_{2} \mathrm{Cl}_{2} \mathrm{~S}$ : C, 38.38; $\mathrm{H}$, 1.84; N, 12.79. Found: C, 38.28; H, 1.95; N, 12.62 .

4,6-Dichloro-2-(dimethylaminoeth-1-yl)benzimidazole hydrochloride (4n): Similar to $\mathbf{4 a}$, from 6 and 2-dimethylaminoethyl chloride hydrochloride. The crude product was purified by column chromatography as described for 4f, and transformed into the hydrochloride as described for 4c: (m.p. $230-233^{\circ} \mathrm{C}, 38 \%$ ); ${ }^{1} \mathrm{H}$ $\mathrm{NMR}\left(\mathrm{D}_{6}-\mathrm{Me}_{2} \mathrm{SO}\right) \delta(\mathrm{ppm}): 2.84\left(\mathrm{~s}, 2 \times \mathrm{CH}_{3}\right)$, 3.46 and 3.69 (2t, $-\mathrm{CH}_{2} \mathrm{CH}_{2}$ ), 7.77 and 7.51 (2d, H-5 and H-7). UV ( $\left.\mathrm{H}_{2} \mathrm{O}, \mathrm{pH} 7\right): 257$ (6700), 291 (12 300), 299 (12600). Elemental analysis: calculated for $\mathrm{C}_{11} \mathrm{H}_{13} \mathrm{~N}_{3} \mathrm{SCl}_{2} \cdot 2 \mathrm{H}_{2} \mathrm{O} \cdot \mathrm{HCl}$ : C, 36.43; H, 5.01; N, 11.58. Found: C, 36.40; $\mathrm{H}, 4.81 ; \mathrm{N}, 11.45$.

4,6-Dichloro-2-(4-nitrobenzylthio)benzimidazole (4o): Similar to $\mathbf{4 a}$, from $\mathbf{6}$ and 4-nitrobenzylchloride (m.p. $132-134^{\circ} \mathrm{C}$ from EtOH/ $\left.\mathrm{H}_{2} \mathrm{O}, 41 \%\right),{ }^{1} \mathrm{H}$ NMR $\left(\mathrm{D}_{6}-\mathrm{Me}_{2} \mathrm{SO}\right) \delta$ (ppm): 4.71 (s, $\mathrm{CH}_{2}$ ), 7.20-8.20 (m, H-arom. and $\mathrm{H}$-benzimidazole). UV $\left(\mathrm{H}_{2} \mathrm{O} / \mathrm{MeOH}, 1: 1\right)$ : 260 (16100), 303 (17800). Elemental analysis: calculated for $\mathrm{C}_{14} \mathrm{H}_{9} \mathrm{~N}_{3} \mathrm{O}_{2} \mathrm{Cl}_{2} \mathrm{~S}$ : C, 47.48; H, 2.57; N, 11.87. Found: C, 47.41; H, 2.39; N, 11.69 .

\section{RESULTS AND DISCUSSION}

\section{Chemical synthesis}

In order to expand the group of benzimidazole derivatives, we synthesised several new benzimidazole ring-containing compounds. In addition to the earlier reported 5,6-dinitro- and 4,6-dinitrobenzimidazoles, their nucleosides and 5,6-dinitro-2-trifluoromethylbenzimidazole $[17,18]$, we have undertaken the synthesis of a series of 1-substituted 5,6-dinitrobenzimidazoles (2a-f) by alkylation of 5,6-dinitrobenzimidazole (1) with appropriate halogenoalkylamine in acetonitrile using 1,8-diazabicyclo[5,4,0]undec-7-en (DBU) as a base. Additionally, 5,6-dinitro-1-(2,3-dihydroxyprop-1-yl)benzimidazole (2g) was obtained in the reaction of $\mathbf{1}$ with glycitole under the same reaction conditions (Scheme 1). The choice of 1-substituents was motivated by their presence in numerous drugs showing a variety of antimicrobial, psychostimulant and other activities.

The second series were sulfur-substituted derivatives of a modified benzimidazole nucleus. The methyl-, chlorine- and carboxy-substituted derivatives of 2-thiobenzimidazoles (3a-m) were S-alkylated with halogenoalkylamines, allyl chloride, or benzyl chloride and its p-nitro and 3,4-dichlorosubstituted derivatives to provide the corresponding derivatives (4a-o). The substrates $\mathbf{3 a - m}$ were previously synthesised by the reaction of carbon disulfide with the corresponding phenylenediamine [6]. The novel 4,6-dichloro-2-thiobenzimidazole (6) was similarly prepared by treating 1,3-dichloro-4,5-diaminobenzene (5) with carbon disulfide (Scheme 2). The resulting derivatives were purified by crystallization or flash column chromatography. In a few cases it was necessary to transform the products into their respective hydrochlorides to obtain a crystalline material. Of the $\mathrm{N}$ - and S-substituted derivatives shown in Schemes 1 and 2 , only $3 \mathbf{c}$ has been previously mentioned in the patent literature [19].

\section{Antibacterial activity}

The antibacterial activity of the benzimidazole derivatives was first tested by the agar disc-diffusion method against Gram-positive and Gram-negative bacteria. The results of these studies are summarized in Table 1. The compounds not shown in the table had no antibacterial activity. The new benzimidazole derivatives reported here, particulary 4d-4n, are approximately as active as metronidazole used as a control substance. In the case of enterobacteria, such as $E$. coli and $P$. vulgaris, the bezimidazoles $\mathbf{4 d}-\mathbf{4 n}$ exhibited moderate activity, whereas metronidazole was inactive. 
<smiles>O=[N+]([O-])c1cc2nc[nH]c2cc1[N+](=O)[O-]</smiles>

1<smiles></smiles>

$2 a-f$

$$
\begin{array}{ll}
\text { 2a } & \mathrm{R}=\mathrm{CH}_{2} \mathrm{CH}_{2} \mathrm{~N}\left(\mathrm{CH}_{3}\right)_{2} \\
\text { 2b } & \mathrm{R}=\mathrm{CH}_{2} \mathrm{CH}_{2} \mathrm{~N}\left(\mathrm{C}_{2} \mathrm{H}_{5}\right)_{2} \\
\text { 2c } & \mathrm{R}=\mathrm{CH}_{2} \mathrm{CH}_{2} \mathrm{CH}_{2} \mathrm{~N}\left(\mathrm{CH}_{3}\right)_{2} \\
\text { 2d } & \mathrm{R}=\mathrm{CH}_{2} \mathrm{CH}_{2} \\
\text { 2e } & \mathrm{R}=\mathrm{CH}_{2} \mathrm{CH}_{2}-\mathrm{O} \\
\text { 2f } & \mathrm{R}=\mathrm{CH}_{2} \mathrm{CH}_{2} \mathrm{OH}
\end{array}
$$<smiles></smiles>

\section{Scheme 1}

On the other hand, metronidazole was more active against bacilli then $\mathbf{2 g}$ and $\mathbf{4 a - f}$ and 4n.

More attention was devoted to the investigation of antibacterial activity against Stenotrophomonas maltophilia strains. S. maltophilia (previously called Pseudomonas maltophilia and Xanthomonas maltophilia) is an increasingly recognized nosocomial pathogen, particularily for immunocompromised patients. Risk factors for S. maltophilia are colonization and infection due to mechanical ventilation, previous exposure to broad spectrum antibiotics, fungal infections, catheterization, prolonged hospitalization and the use of equipment contacting with the respiratory tract, such as nebulizers. S. maltophilia is found in a wide variety of ageratic, soil, and rhizosphere environments and on various contaminated materials and fomites, including faucets, showers, ice-making machines etc. Several of these have been implicated as sources of nosocomial infections with $S$. maltophilia. With the exception of trimetoprim-sulfamethoxazole (Biseptol, Cotrimorazole), many post-therapy isolates of $S$. maltophilia quickly become resistant to antimicrobial agents [20]. The tests presented here (Table 2) show that only compounds $\mathbf{4 d}$, 4e, $4 \mathbf{f}$ and $4 \mathbf{n}$, which are inactive against standard and nosocomial strains of B. cepacia and $P$. aeruginosa, were active against nosocomial strains of $S$. maltophilia. Compounds $\mathbf{4 f}$ and 4n displayed antibacterial activity also 
<smiles>[R]c1cc2[nH]c(S)nc2c([R1])c1[R2]</smiles><smiles>[R16]c1nc2c([R])c([R])c([R3])cc2[nH]1</smiles>

3a-m

4a-m

a $\mathrm{R}_{1}, \mathrm{R}_{2}, \mathrm{R}_{3}=\mathrm{H}, \mathrm{R}_{4}=\mathrm{CH}_{2} \mathrm{CH}_{2} \mathrm{~N}\left(\mathrm{CH}_{3}\right)_{2}$

b $\mathrm{R}_{1}=\mathrm{H}, \mathrm{R}_{2}, \mathrm{R}_{3}=\mathrm{CH}_{3}, \mathrm{R}_{4}=\mathrm{CH}_{2} \mathrm{CH}_{2} \mathrm{~N}\left(\mathrm{CH}_{3}\right)_{2}$

c $\mathrm{R}_{1}, \mathrm{R}_{3}=\mathrm{H}, \mathrm{R}_{2}=\mathrm{Cl}, \mathrm{R}_{4}=\mathrm{CH}_{2} \mathrm{CH}_{2} \mathrm{~N}\left(\mathrm{CH}_{3}\right)_{2}$

d $\mathrm{R}_{1}=\mathrm{H}, \mathrm{R}_{2}, \mathrm{R}_{3}=\mathrm{Cl}, \mathrm{R}_{4}=\mathrm{CH}_{2} \mathrm{CH}_{2} \mathrm{~N}\left(\mathrm{CH}_{3}\right)_{2}$

e $\mathrm{R}_{1}=\mathrm{H}, \mathrm{R}_{2}, \mathrm{R}_{3}=\mathrm{Cl}, \mathrm{R}_{4}=\mathrm{CH}_{2} \mathrm{CH}_{2} \mathrm{~N}\left(\mathrm{C}_{2} \mathrm{H}_{5}\right)_{2}$

f $\mathrm{R}_{1}=\mathrm{H}, \mathrm{R}_{2}, \mathrm{R}_{3}=\mathrm{Cl}, \mathrm{R}_{4}=\mathrm{CH}_{2} \mathrm{CH}_{2} \mathrm{CH}_{2} \mathrm{~N}\left(\mathrm{CH}_{3}\right)_{2}$

g $\mathrm{R}_{1}=\mathrm{H}, \mathrm{R}_{2}, \mathrm{R}_{3}=\mathrm{Cl}, \mathrm{R}_{4}=\mathrm{CH}_{2} \mathrm{CH}_{2}-\mathrm{N} \mathrm{O}$

h $\quad \mathrm{R}_{1}=\mathrm{H}, \mathrm{R}_{2}, \mathrm{R}_{3}=\mathrm{Cl}, \mathbf{R}_{4}=p$-nitrobenzyl

i $\mathrm{R}_{\mathrm{l}}=\mathrm{H}, \mathrm{R}_{2}, \mathrm{R}_{3}=\mathrm{Cl}, \mathrm{R}_{4}=\mathrm{CH}_{2}-\mathrm{Cl}$

j $\quad \mathrm{R}_{1}, \mathrm{R}_{3}=\mathrm{H}, \mathrm{R}_{2}=\mathrm{COOH}, \mathrm{R}_{4}=\mathrm{CH}_{2} \mathrm{CH}=\mathrm{CH}_{2}$

k $\quad \mathrm{R}_{1}, \mathrm{R}_{3}=\mathrm{H}, \mathrm{R}_{2}=\mathrm{COOH}, \mathrm{R}_{4}=$ benzyl

I $\mathbf{R}_{1}, \mathbf{R}_{3}=\mathrm{H}, \mathbf{R}_{2}=\mathrm{COOH}, \mathbf{R}_{4}=p$-nitrobenzyl

m $\mathrm{R}_{1}, \mathrm{R}_{3}=\mathrm{H}, \mathrm{R}_{2}=\mathrm{COOH}, \mathrm{R}_{4}=\mathrm{CH}_{2}-\mathrm{Cl}$<smiles>Nc1cc(Cl)cc(Cl)c1N</smiles><smiles>Sc1nc2c(Cl)cc(Cl)cc2[nH]1</smiles>

6<smiles>[R10]c1nc2c(Cl)cc(Cl)cc2[nH]1</smiles>

4n $\mathrm{R}=\mathrm{CH}_{2} \mathrm{CH}_{2} \mathrm{~N}\left(\mathrm{CH}_{3}\right)_{2}$

40

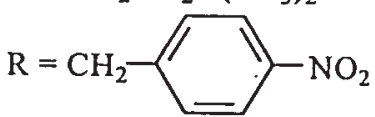

Scheme 2

against the standard strain of $S$. maltophilia NCTC 13637 (MIC 400 and $200 \mu \mathrm{g} / \mathrm{ml}$, respectively). Derivatives $\mathbf{4 d}$ and $\mathbf{4 e}$ - inactive against standard strains of Pseudomonas family - are active against all of nosocomial strains of $S$. maltophilia (MIC $50-400 \mu \mathrm{g} / \mathrm{ml}$ ) tested here. In contrast, compounds $\mathbf{4 f}$ and 4n moderately active against standard strains, were inactive against 49 and 16 nosocomial strains.
The results obtained indicate that the compounds studied here or their possible structural analogues could be potential chemotherapeutics in the case of $S$. maltophilia infection. It is noteworthy that the nosocomial $S$. maltophilia strains examined here were resistant to $\beta$-lactam antibiotics, including penems, azeotronams and cephalosporins of the 3rd generation and showed considerable resistance to aminoglycosides and quinolones. 
Table 1. Antimicrobial activity of substituted benzimidazoles against Gram-positive and Gram-negative bacteria strains

\begin{tabular}{|c|c|c|c|c|c|c|c|c|c|}
\hline \multirow{3}{*}{ Bacteria strain } & \multicolumn{9}{|c|}{ Diameter of growth inhibition area (mm) } \\
\hline & \multicolumn{9}{|c|}{ Compound } \\
\hline & $2 \mathrm{~g}$ & $4 \mathbf{a}$ & $4 \mathbf{b}$ & $4 c$ & $4 d$ & $4 e$ & $4 \mathbf{f}$ & $4 n$ & $\begin{array}{l}\text { Metroni- } \\
\text { dazole }\end{array}$ \\
\hline S. aureus ATCC 6538P & 0 & trace & trace & 0 & 12 & 12 & 14 & 15 & 11 \\
\hline S. aureus ATCC 25923 & trace & 11 & trace & trace & 12 & 14 & 12 & 14 & 12 \\
\hline S. aureus NCTC 4163 & 0 & trace & 0 & 0 & 11 & 12 & 12 & 11 & 11 \\
\hline M. flavus NCIB 8166 & 12 & 11 & trace & trace & trace & 0 & 10 & 10 & 12 \\
\hline B. subtilis ATCC 6633 & 17 & 11 & 0 & 11 & 11 & 11 & 12 & 14 & 32 \\
\hline B. stenothermophilus ATCC 7953 & 14 & 11 & 0 & 12 & 11 & 13 & 13 & 12 & 30 \\
\hline B. cereus ML 98 & 14 & trace & trace & 11 & 12 & 13 & 12 & 14 & 15 \\
\hline E. coli NCTC 8196 & 0 & 0 & 0 & 0 & 11 & 11 & 12 & 12 & 0 \\
\hline P. vulgaris NCTC 4635 & 0 & trace & trace & trace & 13 & 11 & 12 & 12 & 0 \\
\hline
\end{tabular}

\section{Anti-protozoal activity}

Drugs (0.1 M) dissolved in dimethyl formamide (DMF) were used in microtitre plate susceptibility assays as previously described [16]. In a few cases (2c, $4 \mathbf{4 f}, \mathbf{4 c}, \mathbf{4 n})$ the compounds required mild heat for dissolution. $4 \mathbf{e}$ was soluble at $10 \mathrm{mM}$ and $\mathbf{4 h}$ crystallized in the assays at $>100 \mu \mathrm{M}$. MIC values for $\mathbf{1}$, $\mathbf{2 a}-\mathbf{2 g}$ and $4 \mathbf{a}-\mathbf{o}$ were estimated as previ- ously described and only compounds with MICs $<100 \mu \mathrm{M}$ in any assay are presented in Table 2. The MIC values for metronidazole $(\mathrm{Mz})$ and albendazole (Alb) are also presented. While neither of the compounds was uniformly as effective as metronidazole, or as effective as albendazole against Giardia, several compounds had MIC values which should encourage further development as lead compounds in a search for new anti-anaerobic

Table 2. Drug susceptibility assay of Pseudomonas family bacteria

\begin{tabular}{|c|c|c|c|c|}
\hline \multirow{3}{*}{ Bacteria strain } & \multicolumn{4}{|c|}{ MIC values (mg/ml) } \\
\hline & \multicolumn{4}{|c|}{ Compound } \\
\hline & 4d & $4 e$ & 4f & $4 n$ \\
\hline S. maltophilia ATCC 13637 & $>400$ & $>400$ & 400 & 200 \\
\hline B. серасіа ATCC 25416 & $>400$ & $>400$ & $>400$ & $>400$ \\
\hline P. aeruginosa ATCC 27863 & $>400$ & $>400$ & $>400$ & $>400$ \\
\hline P. aeruginosa ATCC 15442 & $>400$ & $>400$ & $>400$ & $>400$ \\
\hline P. aeruginosa NCTC 6749 & $>400$ & $>400$ & $>400$ & $>400$ \\
\hline $\begin{array}{l}\text { S. maltophilia } 67 \text { clinical } \\
\text { strains }\end{array}$ & $\begin{array}{l}100 \\
-3 \text { strains } \\
100-200 \\
-35 \text { strains } \\
300-400 \\
\quad-29 \text { strains }\end{array}$ & $\begin{array}{l}50 \\
-2 \text { strains } \\
100-200 \\
-28 \text { strains } \\
300-400 \\
-37 \text { strains }\end{array}$ & $\begin{array}{l}100 \\
-2 \text { strains } \\
200 \\
-4 \text { strains } \\
400 \\
-12 \text { strains } \\
>400 \\
-49 \text { strains }\end{array}$ & $\begin{array}{l}100 \\
-2 \text { strains } \\
200 \\
-5 \text { strains } \\
400 \\
-44 \text { strains } \\
>400 \\
-16 \text { strains }\end{array}$ \\
\hline
\end{tabular}

Metronidazole used as control substance; MIC > $400(\mu \mathrm{g} / \mathrm{ml})$; replica plates method was employed. 
Table 3. Drug susceptibility assays of G. duodenalis, T. vaginalis and E. histolytica tested against metronidazole $(\mathrm{Mz})$, albendazole $(\mathrm{Alb})$ and new benzimidazole derivatives.

Values are presented as MIC $(\mu \mathrm{M})$.

\begin{tabular}{|c|c|c|c|c|c|c|c|c|}
\hline \multirow{2}{*}{ Drug } & \multicolumn{5}{|c|}{ G. duodenalis } & \multicolumn{2}{|c|}{ T. vaginalis } & E. histolytica \\
\hline & WB & $\mathrm{WB} / \mathrm{Alb}$ & WB-M1 & 106 & $2 \mathrm{ID}_{10}$ & F1623 & F1623-M1 & MU TM \\
\hline $\mathrm{Mz}$ & 6.3 & 6.3 & 50 & 6.3 & 50 & 1.6 & 25 & 25 \\
\hline $\mathrm{Alb}$ & 1.6 & 100 & 3.2 & 1.6 & ND & $>200$ & $>200$ & $>200$ \\
\hline $\mathbf{1}$ & 200 & ND & ND & 200 & ND & 50 & $>200$ & 200 \\
\hline $\mathbf{2 a}$ & $>200$ & $\mathrm{ND}$ & $\mathrm{ND}$ & $>200$ & ND & 25 & $>200$ & 100 \\
\hline $2 b$ & 100 & 200 & ND & 200 & ND & 25 & $>200$ & 200 \\
\hline $2 c$ & $>200$ & ND & ND & $>200$ & ND & 50 & $>200$ & $>200$ \\
\hline $2 d$ & 200 & ND & ND & 200 & ND & 25 & $>100$ & $>200$ \\
\hline $2 e$ & $>200$ & ND & ND & $>200$ & ND & 50 & $>200$ & 200 \\
\hline $2 f$ & 200 & $\mathrm{ND}$ & $\mathrm{ND}$ & 200 & $\mathrm{ND}$ & 100 & $>200$ & $>200$ \\
\hline 41 & 100 & ND & 100 & 100 & 100 & 100 & 100 & ND \\
\hline $4 \mathrm{~h}$ & 25 & 25 & 25 & 25 & 25 & 100 & 100 & 25 \\
\hline 40 & 50 & 50 & 50 & 50 & 50 & 50 & $>200$ & 50 \\
\hline
\end{tabular}

${ }^{*} \mathrm{ND}$, not determined.

protozoal clinical agents. Compounds $\mathbf{2 a}, \mathbf{2 b}$, $\mathbf{2 c}$, and to a lesser extent $\mathbf{2 e}$, were the most effective against $T$. vaginalis. These 5,6-dinitro-1-(aminoethyl)benzimidazoles may act via reduction of the nitro group via ferredoxin in the same way metronidazole acts, but not as inhibitors of tubulin polymerization as albendazole does, since the metronidazole-resistant line F1623-M1 was not susceptible to these compounds. This is what is expected of drugs with a similar mechanism of action to metronidazole.

The compound $\mathbf{4 h}$ and to a lesser extent 40 were the most effective against Giardia and Entamoeba. While 5,6-dichloro and 4,6-dichloro benzimidazoles carry a nitrobenzyl group, the mechanism of action may not be via the nitro group, since the metronidazole-resistant Giardia lines and the parent isolates were similarly susceptible. In addition, the MIC of $\mathbf{4 h}$ against Entamoeba was the same as that of metronidazole. Compound 41 which also carries the nitrobenzyl group but is a 5-carboxybenzimidazole, rather than a dichloro-benzimidazole, was ineffective in all assays suggesting that the nitrobenzyl group was not the all-important functional moiety in 4h and 4o. Since benzimidazole compounds have not previously been shown to be effective against Entamoeba and in our hands albendazole is ineffective against Trichomonas (Table 3), our results, particularly those for $\mathbf{4 h}$, are worth pursuing in the search for new effective anti-protozoal drugs.

We would like to thank Raymond Campbell for his expert technical assistance in carrying out the drug susceptibility assays

\section{R E F E R E N C E S}

1. Sheehan, D.J., Hitchcock, C. A. \& Sibley, C.M. (1999) Current and emerging antifungal agents. Clin. Microbiol. Rev. 12, 40-79.

2. Habib, N.S., Soliman, R., Ashour, F.A. \& el-Taiebi, M. (1997) Synthesis and antimicrobial testing of novel oxadiazolylben- 
zimidazole derivatives. Pharmazie 52 , $746-749$.

3. Tuncbilek, M., Goker, H., Ertan, R., Eryigit, R., Kendi, E. \& Altanlar E. (1997) Synthesis and antimicrobial activity of some new anilino benzimidazoles. Arch. Pharm. 330, 372-376.

4. Pedini, M., Alunni Bistochi, G., Ricci, A., Bastianini, L. \& Lepri, E. (1994) New heterocyclic derivatives of benzimidazole with germicidal activity - XII. Synthesis of N1-glycosyl-2-furyl benzimidazoles. Farmaco 49, 823-827.

5. Lackner, T.E. \& Clissold, S.P. (1989) Bifonazole. A review of its antimicrobial activity and therapeutic use in superficial mycoses. Drugs 38, 204-225.

6. Devivar, R.V., Kawashima, E., Revankar, G.R., Breitenbach, J., Kreske, E., Drach, J. \& Townsend, L. (1994) Benzimidazole ribonucleosides: Design, synthesis, and antiviral activity of certain 2-(alkylthio)- and 2-(benzylthio)-5,6-dichloro-1-( $\beta$-D-ribofuranosyl)benzimidazoles. J. Med. Chem. 37, 2942-2949.

7. Pawełkiewicz, J. \& Zodrow, K. (1957) Prekursory biosyntezy nukleotydocyjanokobalamin. Acta Microbiol. Polon. 6, 9-15 (in Polish).

8. Burton, D.E., Lambie, A.J., Ludgate, J.C., Newbold, G.T., Percival, A. \& Saggers, D.T. (1965) 2-Trifluoromethylbenzimidazoles; a new class of herbicidal compounds. Nature 208, 1166-1170.

9. Navarette-Vazquez, G., Cedillo, R., Hernandez-Campos, A., Yepez, L., Hernandez-Luis, F., Valdez, J., Morales, R., Cortes, R., Hernandez, M. \& Castillo, R. (2001) Synthesis and antiparasitic activity of 2-(trifluoromethyl)benzimidazole derivatives. Bioorg. Med. Chem. Lett. 11, 187-190.

10. Xiao, L., Saeed, K. \& Herd, R.P. (1996) Efficacy of albendazole and fenbedazole against
Giardia infection in cattle. Vet. Parasitol. 61, 165-170.

11. Katiyar, S.K., Gordon, V.R., McLaughlin, G.L. \& Edlind, T.D. (1994) Antiprotozoal activities of benzimidazoles and correlations with beta-tubulin sequence. Antimicrob. Agents Chemother. 38, 2086-2090.

12. Bishop, B.C., Chelton, E.T.J. \& Jones, A.S. (1964) The antibacterial activity of some fluorine-containing benzimidazoles. Biochem. Pharmacol. 13, 751-754.

13. Stefańska, J.Z., Gralewska, R., Starościak, B.J. \& Kazimierczuk, Z. (1999) Antimicrobial activity of substituted azoles and their nucleosides. Pharmazie 54, 879-884.

14. National Committee for Clinical Laboratory Standards, NCCLS Approval Standard Document M2-A7. (2000) Villanova, PA, U.S.A.

15. National Committee for Clinical Laboratory Standards, NCCLS Approval Standard Document M7-A5. (2000) Villanova, PA, U.S.A.

16. Upcroft, J.A. \& Upcroft, P. (2001) Drug susceptibility testing of the anaerobic protozoa. Antimicrob. Agents Chemother. 45, 18101814.

17. Kazimierczuk, Z. \& Shugar, D. (1989) Preparation and properties of the 5,6- and 4,6-dinitro derivatives of benzimidazole and their 1- $\beta$-D-ribofuranosides. Nucleosides Nucleotides 8, 1379-1385.

18. Büchel, K.-H. (1970) Herbizide Trifluoromethyl-benzimidazole. Z. Naturforsch. 25b, 934944.

19.Jpn. Patent 1097861, through Chem. Abstr. (1963) 58, 13964.

20. Alonso, A. \& Martinez, J.C. (1997) Multiple antibiotic resistance in Stenotrophomonas maltophilia. Antimicrob. Agents Chemother. 41, 1140-1142. 